cians and patients think and talk about overuse or misuse of health care resources.

The AAFP's list consists of the following 5 recommendations:

1. Do not do imaging for low back pain within the first 6 weeks, unless red flags are present. Red flags include, but are not limited to, severe or progressive neurological deficits or when serious underlying conditions such as osteomyelitis are suspected. Imaging of the lower spine before 6 weeks does not improve outcomes, but does increase costs and involves unnecessary radiation exposure. Low back pain is the 5 th most common reason for all physician visits.

2. Do not routinely prescribe antibiotics for acute mild-to-moderate sinusitis unless symptoms last for 7 or more days or symptoms worsen after initial clinical improvement. Symptoms must include discolored nasal secretions and facial or dental tenderness when pressure is applied. Most sinusitis in the ambulatory setting is due to a viral infection that will resolve on its own. Despite consistent recommendations to the contrary, antibiotics are prescribed in more than $80 \%$ of outpatient visits for acute sinusitis, resulting in risk of side effects without benefit. Sinusitis accounts for 16 million office visits and $\$ 5.8$ billion in annual health care costs.

3. Do not use dual-emission X-ray absorptiometry (DEXA) in women aged younger than 65 years or men aged younger than 70 years with no risk factors. DEXA is not cost-effective in younger, low-risk patients but is cost-effective in older patients.

4. Do not order electrocardiograms or other cardiac screening for low-risk patients without symptoms. There is little evidence that detection of coronary artery stenosis in asymptomatic patients at low risk for coronary heart disease improves health outcomes. False-positive tests are likely to lead to harm through unnecessary invasive procedures, overtreatment, and misdiagnosis. Potential harms of this routine annual screening exceed the potential benefit.

5. Do not perform Pap smears on women aged younger than 21 years or who have had a hysterectomy for noncancer disease. Most observed abnormalities in adolescents regress spontaneously; therefore, Pap smears for this age-group can lead to unnecessary anxiety, additional testing, and cost. Pap smears are not helpful in women after hysterectomy (for noncancer disease), and there is little evidence for improved outcomes.

The lists drawn up by the campaign's 8 other medical specialty partners are available on the Choosing Wisely website. In addition, 8 more medical specialty organizations signed on to the campaign during the
April 2012 press event. They are scheduled to release their lists this fall.

In an interview after the press event, Stream stressed the need to develop a solid, secure, physicianpatient relationship so meaningful patient conversations can take place.

"People really do need a doctor who knows them and can help them navigate the medical system if they have a serious medical problem," he said. "It is also important to note that it is one thing to get a 'Choosing Wisely' decision from a doctor who knows you, but that to do that, you have to build trust up over time.

"I think that as family physicians, our role is unique, because we are not only managing the care that we give, but it is also just as critical that we coordinate care for our patients using our subspecialty colleagues."

Matt Brown

AAFP News Now

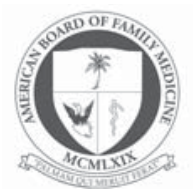

From the American

Board of Family Medicine

Ann Fam Med 2012;10:374-375. doi:10.1370/afm.1423.

\section{ABFM'S HEART FAILURE SELF-ASSESSMENT MODULE SIMULATION ACTIONS VIS-À-VIS GUIDELINE RECOMMENDATIONS}

The American Board of Family Medicine (ABFM) introduced Maintenance of Certification for Family Physicians (MC-FP) in 2004 in response to policy adopted by the American Board of Medical Specialties (ABMS.) ${ }^{1}$ ABFM reported in 2006 the initial Diplomate experiences with MC-FP. ${ }^{2}$ At that time, ABFM had SelfAssessment Modules (SAMs), consisting of a 60 -item knowledge assessment followed by a virtual patient clinical simulation available only for hypertension, type 2 diabetes mellitus, asthma, and depression. Since that time, ABFM has deployed modules for coronary artery disease, chronic heart failure, well child care, maternity care, preventive care, care of the vulnerable elderly, pain management, early childhood illness, cerebrovascular disease, and health behavior. Each of the SAMs includes a Diplomate assessment of both the knowledge assessment and the simulation components.

In addition to the Diplomates' subjective assessments of the SAMs, ABFM captures the actions taken during each simulation, including the action itself, the simulated date and time of the action, and the simulated patient's current health state. This information is 
captured in an action $\log$, which serves as a persistent record of the Diplomate's traversal through the simulation scenario.

ABFM introduced the chronic heart failure (CHF) SAM and associated simulation in 2006. We present in Figure 1 a graphical summary of simulation actions taken in the CHF SAM, as extracted from the simulation action logs from 2006 to 2011 . We present the results as percentage of simulations in which given actions occurred.

The results indicate overall high use of angiotensin converting inhibitors (ACEInhibitors), ACE inhibitors and/or angiotensin receptor blockers (ACEInhibitorsARBS), but surprisingly low use of beta-adrenergic blocking agents (BetaBlockers.) Digitalis preparations (DigitalisPrep) demonstrated very low use. The majority of Diplomates also did a formal assessment of left ventricular function (Echocardiogram.)

The CHF simulations generally present scenarios representing patients with stage $\mathrm{C}$ heart failure, as defined in the 2009 ACCF/AHA heart failure guideline. ${ }^{3}$ According to that guideline, ACE inhibitors and beta-adrenergic blockers should routinely be used in these patients. ${ }^{3}$ This discrepancy between recommended therapy and the low percentage of beta-adrenergic blocker prescriptions in the simulations suggests that the knowledge assessments should perhaps place greater emphasis on the use of these agents in class $\mathrm{C}$.heart failure. The current $\mathrm{CHF}$ knowledge assessment contains only 7 items that reference beta-blockers, and nearly all of these present a beta-adrenergic agent only as historical information in the clinical stem of the item, rather than as a focus of decision-making in the item.

These results suggest that the simulations, although clearly presenting a virtual patient environment, can serve as a useful probe for identifying possible management gaps that should be emphasized in the SAM knowledge assessments.

Michael D. Hagen, MD

\section{References}

1. American Board of Medical Specialties. ABMS Maintenance of Certification. 2006 http://www.abms.org/Maintenance_of_Certification/ ABMS_MOC.aspx. Accessed Jul 29, 2011.

2. Hagen MD, Ivins D, Puffer JC, Rinaldo J, Roussel GH, Sumner W, $\mathrm{Xu}$ J. Maintenance of certification for family physicians (MC-FP) SelfAssessment Modules (SAMs): the first year. JABFM. 2006;19:398-403.

3. Jessup M, et al. 2009 focused update: ACCF/AHA guidelines for the diagnosis and management of heart failure in adults. Circulation. 2009;119:1977-2016.

\section{Figure 1. Chronic heart failure Self-Assessment Module simulations including indicated actions.}

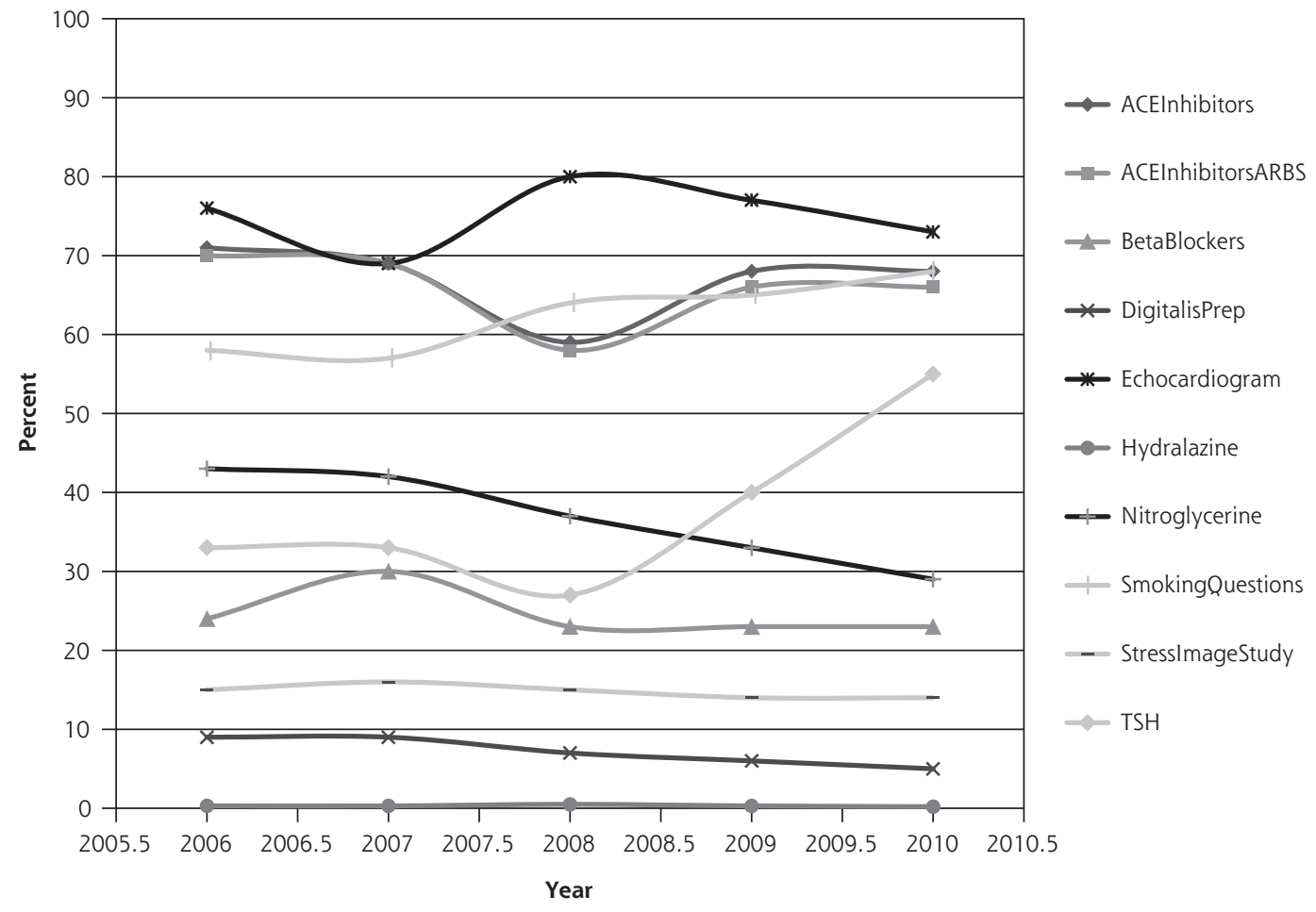

ACEInhibitors = angiotensin converting inhibitors; ACEInhibitorsARBS $=$ ACE inhibitors and/or angiotensin receptor blockers; BetaBlockers $=$ beta-adrenergic-blocking agents; DigitalisPrep = digitalis preparations; TSH = thyroid-stimulating hormone. 